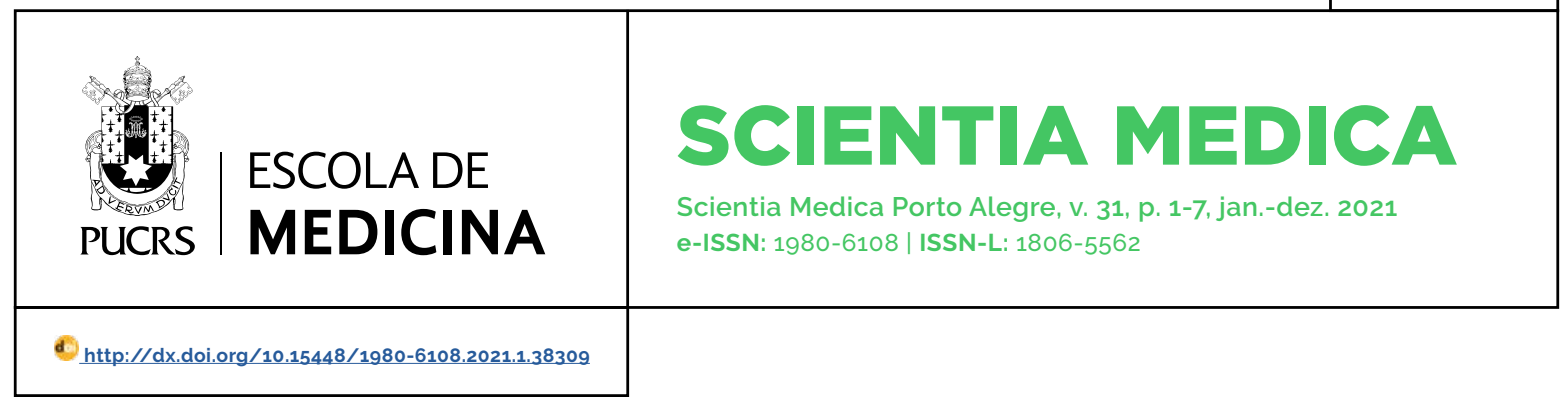

EDUCACIÓN EN CIENCIAS DE LA SALUD

\title{
Teoría sindémica como un enfoque para explicar la interacción entre problemas de salud pública
}

\author{
A teoria sindêmica como abordagem para explicar a interação entre problemas de \\ saúde pública \\ Syndemics framework as an approach to explain the interaction between public health \\ problems
}

Silvia Quiroz Mena ${ }^{1}$

orcid.org/0000-0002-5442-3872

silvia.quiroz@udea.edu.co

\section{Wilson Cañon \\ Montañez ${ }^{1}$}

orcid.org/0000-0003-0729-5342

wilson.canon@udea.edu.co

Recibido: 29/05/2020.

Aceptado: 03/10/2020

Publicado: 25/02/2021

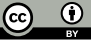

Artigo está licenciado sob forma de uma licença Creative Commons Atribuição 4.0 Internacional.
Resumen: La teoria sindémica proporciona un marco innovador que permite comprender ¿por qué ciertos problemas de salud pública se concentran geográfica y temporalmente? y ¿cómo estos problemas interactúan biológica y socialmente?, generando efectos nocivos en grupos vulnerables. Sin embargo, la evidencia empírica que pone a prueba esta teoría aún es escasa y la existente tiene ciertas limitaciones en el enfoque y medición de conceptos claves. Por este motivo, este artículo tiene como propósito generar una discusión sobre algunas implicaciones conceptuales y metodológicas del uso de la teoria sindémica. El correcto abordaje de la teoría sindémica permite direccionar futuras investigaciones en este tema y promueve su importancia para el estudio de problemas de salud en el contexto de Latinoamérica.

Palabras clave: Sindémia, salud pública, prueba de estudio conceptual, métodos.

Resumo: A teoria sindêmica fornece um modelo inovador que nos permite entender por que determinados problemas de saúde pública estão geograficamente e temporalmente concentrados e como esses problemas interagem biológica e socialmente, gerando efeitos nocivos sobre grupos vulneráveis. No entanto, as evidências empíricas que testam essa teoria ainda são escassas e a existente apresenta certas limitações na abordagem e mensuração de conceitos-chave. Por esse motivo, este artigo tem como objetivo gerar uma discussão sobre algumas implicações conceituais e metodológicas do uso da teoria sindêmica. A correta abordagem da teoria sindêmica permite direcionar pesquisas futuras sobre o tema e promove sua importância para o estudo dos problemas de saúde no contexto da América Latina.

Palavras-chave: Sindemia, saúde pública, teste de estudo conceitual, métodos

Abstract: The syndemics theory provides an innovative framework that allows to understand why some public health problems are geographically and temporarily concentrated?, and how these problems interact biologically and socially? generating harmful effects on vulnerable groups. However, the empirical evidence that supports this theory is still scarce and the existing has certain limitations in the approach and measurement of key concepts. For this reason, this article aims to generate a discussion about some conceptual and methodological implications of the use of syndemics theory. The correct approach to syndemics theory allows directing future research on this topic and promotes its importance for the study of health problems in the Latin American context.

Keywords: Syndemic, public health, proof of concept study, methods.

ABREVIATURAS: SAVA, substance abuse, violence and AIDS; SIDA, sindrome de inmunodeficiencia adquirida; VIH, virus de la inmunodeficiencia humana; IECA, inhibidores de la enzima convertidora de angiotensina; DAG, Directed Acyclic Graph. 


\section{INTRODUCCIÓN}

La teoría sindémica se ha convertido en un fuerte referente a la hora de comprender la interacción o coexistencia de dos o más enfermedades [1-5]. Esto va más allá de la idea de co-morbilidad, es decir, existen diferencias que no son simplemente semánticas. Para explicar mejor, el termino co-mórbido se centra en los límites nosológicos y la superposición de diagnósticos clínicos, mientras que la sindémia se enfoca en los colectivos vulnerables que experimentan enfermedades, epidemias o problemas de salud pública concurrentes, aumentado las consecuencias negativas en la salud [3].

El concepto de sindémia fue introducido inicialmente por la antropología médica, pero ha ganado reconocimiento en otros campos de la salud [2]. La primera sindémia descrita en 1996, se conoció como SAVA (substance abuse, violence and AIDS, por su sigla en inglés), y postuló la interacción de tres eventos en salud, abuso de sustancias, violencia y sindrome de inmunodeficiencia adquirida (SIDA), como condiciones interdependientes que coexisten en el cuerpo humano y en la vida social en entornos urbanos de bajos ingresos económicos [6]. Posteriormente, surgieron otros estudios que analizaron la sindémia entre enfermedades infecciosas en contextos y condiciones de vida particulares. Por ejemplo, el llamado circulo vicioso de interacción entre malaria y virus de la inmunodeficiencia humana $(\mathrm{VIH})$ en mujeres africanas [3].

En la actualidad, esta teoria ha sido aplicada a diferentes problemas en salud bajo diversos métodos; tal y como lo muestra Tsai et al., [7] en una revisión sistemática publicada en 2017, cuyo objetivo fue analizar críticamente estudios empíricos sobre sindémia e introducir conceptos claves para medir sus efectos en salud poblacional. En esta revisión, se seleccionaron 71 estudios observacionales entre el 2003 al 2016, encontrándose que más del $50 \%$ incluyeron el VIH como resultado de interés y otros se concentraron en temáticas relacionadas con la salud mental (8\%) y el uso de sustancias psicoactivas (8\%).
Con respecto al diseño metodológico de los estudios, el 79\% eran de tipo transversal y el 21\% con abordaje o medición longitudinal.

Los autores de esta revisión hacen una critica importante sobre la forma como se asumen y se abordan conceptos claves a la hora de aplicar la teoría sindémica. Por ejemplo, la "interacción" es uno de los conceptos que menor apoyo empírico ha tenido en comparación con otros. Esto se vincula con retos metodológicos en términos de su medición y análisis, es decir, la mayoría de los estudios cuantitativos incluidos dentro de la revisión no lograron evidenciar si realmente lo evaluado se correspondia con epidemias interactuantes o mutuamente causales. Además, se concentraron en asociaciones meramente aditivas, más que en pruebas formales de interacción [7].

En este orden, el presente articulo tiene como propósito discutir algunas implicaciones conceptuales y metodológicas derivadas de la aplicación de la teoría sindémica e introducir algunas reflexiones considerando problemas de salud pública relevantes para el contexto de Latinoamérica, en donde se evidencia un escaso conocimiento empirico sobre esta teoria.

\section{Implicaciones conceptuales de la teoría sindémica}

La aplicación de esta teoría intenta responder: ¿por qué ciertos problemas de salud se agrupan o se concentran?, ¿cuáles son los mecanismos a través de los cuales interactúan biológicamente en los individuos y grupos?, ¿cómo la interacción negativa afecta el curso de la trayectoria de cada problema de salud? y ¿cómo las fuerzas sociales contribuyen al agrupamiento e interacción de dichos problemas? [2,3].

No obstante, la evidencia empírica ha tenido ciertas limitaciones a la hora de definir los conceptos claves detrás de las respuestas a estas preguntas. El primer concepto importante es el de concentración de la enfermedad, el cual hace referencia a cómo la carga de dos o más epidemias se concentra geográfica y temporalmente [3]. Por ejemplo, desde 1975 hasta 2015, África subsahariana y el Sur de Asia han tenido prevalencias de hipertensión mayores 
al 30\% tanto en hombres como en mujeres [8], al tiempo que han sido regiones endémicas para malaria, concentrando el $80 \%$ de la carga mundial [9]. Aquí es clave resaltar que el contexto social y ambiental de ambas regiones podría jugar un papel relevante en la concentración de dichas enfermedades.

¿Esto es suficiente para probar la teoría sindémica?, es decir, ¿se podría indicar que la hipertensión y la malaria son eventos sindémicos en África y en el Sur de Asia?

Estas preguntas reflejan una discusión importante, dado que si bien, un análisis de la concentración de enfermedades por si solo le da valor a la teoría sindémica, esto no es suficiente [3]. Por tanto, hay que incluir la "interacción" como un segundo concepto clave para probar esta teoria, ya que la co-existencia o co-ocurrencia de enfermedades no indican necesariamente que estén asociadas entre sí.

La interacción en el contexto de la teoría sindémica hace referencia a los diferentes mecanismos a través de los cuales las enfermedades y otras condiciones de salud se relacionan biológicamente dentro de los cuerpos individuales, multiplicando la carga de salud general en las poblaciones [1-3]. Se han planteado algunas formas de interacción, por un lado, unidireccional, es decir, "los cambios en el cuerpo causados por una enfermedad pueden promover el éxito de otra enfermedad" [2]. Por ejemplo, la exposición a malaria se ha asociado con mayor probabilidad de hipertensión en adultos africanos [10], esto se ha explicado por algunos mecanismos biológicos, tales como la modulación de respuestas inflamatorias por el proceso infeccioso que lleva a rigidez vascular; la afectación del flujo sanguíneo por exposición a la malaria durante la gestación, entre otros [10-12].

Por otro lado, también se habla de interacción bidireccional, en donde, las trayectorias de ambas enfermedades pueden verse afectada de manera conjunta, ya sea, por asuntos fisiopatológicos, por ejemplo, la infección por VIH facilita la adquisición de la malaria. A su vez, la reacción inmune contra la malaria hace que las personas sean dos veces más infecciosas para el VIH. "Por lo tanto, el VIH contribuye al desarrollo de malaria y la malaria contribuye al VIH" [3].

Estas trayectorias también pueden afectarse por el manejo clínico de alguna de las enfermedades, lo que se conoce como sindémia iatrogénica [3]. Un buen ejemplo es la reciente evidencia sobre precauciones en el uso de antihipertensivos como los Inhibidores de la Enzima Convertidora de Angiotensina (IECA) en pacientes de zonas endémicas para malaria, ya que se ha reportado un efecto protector entre altos niveles de angiotensina II y la patogénesis de la malaria cerebral [13]. Sin embargo, el análisis de esta evidencia debe ser cuidadoso a luz de determinar si dicha asociación es o no causal considerando modelos de causalidad como los criterios de Hill, especificamente en términos de fuerza y consistencia de los hallazgos. No obstante, es importante mencionar que las técnicas estadisticas comúnmente usadas ofrecen medidas de asociación, no precisamente de efecto causal. Recientemente y como alternativas para el estudio de la causalidad, algunos autores de la epidemiología moderna desarrollaron nuevas herramientas metodológicas para evaluación de la inferencia causal [14].

La teoría sindémica, explora más allá de estos mecanismos biológicos, dado que es bien reconocido que las enfermedades se desarrollan dentro de los contextos sociales de quienes las padecen [3]. Por ello, un tercer concepto clave, es el de las fuerzas sociales a gran escala como determinantes de la sindémia. Esto no es un asunto nuevo, ya desde 1995, la teoría de las causas fundamentales expuesta por Phelan et al, abordaba esta idea de cómo factores sociales relacionados con la distribución del poder, el prestigio y los ingresos económicos condicionan el riesgo de enfermar y morir [15]. Dentro del marco sindémico se hace mayor énfasis en los entornos sociales construidos y las condiciones ambientales que como se mencionó contribuyen enormemente tanto a la agrupación de enfermedades como a la interacción [3].

Comprender los conceptos expuestos (concentración de la enfermedad, la interacción y el papel de las fuerzas sociales) de manera 
integral ha sido una gran limitación en la aplicación de esta teoría. Asimismo, como la definición de hipótesis con el interés de valorar posibles variables confusoras, de interacción o de modificación del efecto, mediadoras y de tipo collider, como un asunto previo a la definición de métodos. Esto puede estar relacionado con la escasa literatura alrededor del tema.

Se sugiere, el uso de herramientas de representación gráfica o diagramación de efectos causales, que permitan visualmente identificar las relaciones causales y roles entre las variables de estudio [16], como se muestra un ejemplo hipotético en la Figura 1. Cabe resaltar, que esto no es un asunto menor a la hora de comprender las sindémias, dado la complejidad de las relaciones a estudiar, sin embargo, hay que considerar que, aunque para este ejercicio fue útil la elaboración de un Gráfico Acíclico Dirigido (Directed Acyclic Graph - DAG, de su sigla en inglés), en otros casos, se requieren de otro tipo de diagramas que permitan observar elementos importantes de la teoría, tales como los efectos de bi-direccionalidad. Se resalta que en la Figura 1 también existe la necesidad de considerar todo el espectro de variables que pueden influenciar relaciones causales entre las enfermedades estudiadas, tales como, factores genéticos, comportamentales, variables no medidas, entre otras.

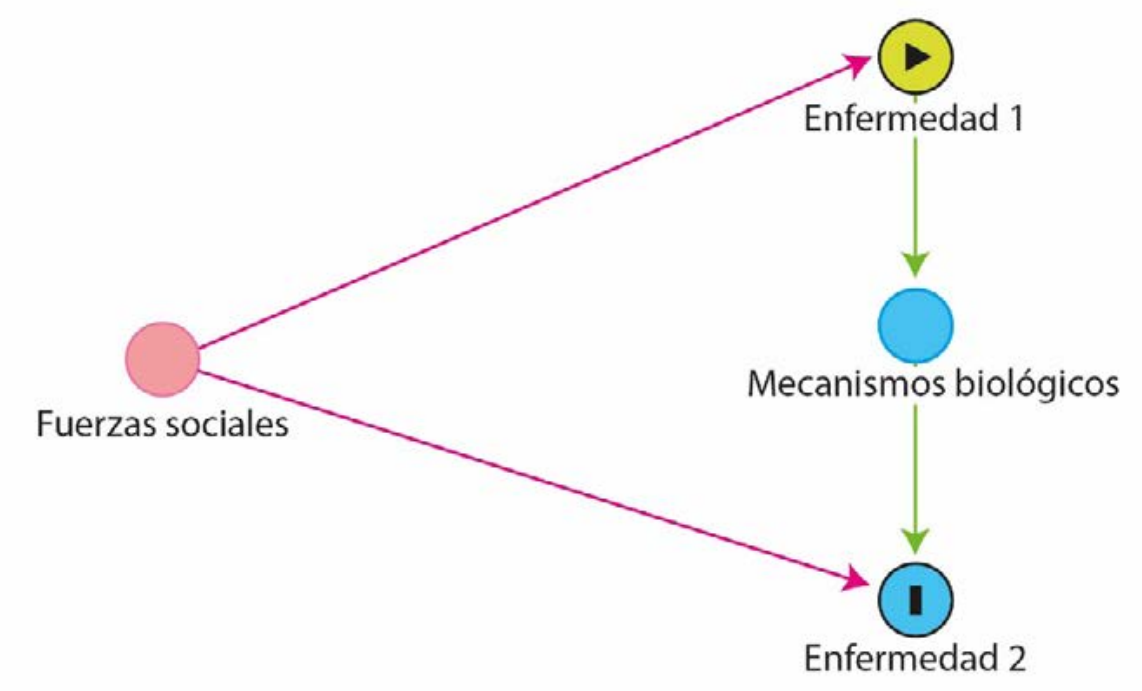

Exposición $\bigcirc$ Desenlace Variable de confusión $\bigcirc$ Variable mediadora

Figura 1 - Ejemplo de gráfico acíclico dirigido en la aplicación de la teoría sindémica

\section{Implicaciones metodológicas de la teoría sindémica}

Para lograr que el abordaje metodológico cumpla con los fundamentos de la teoría sindémica es importante considerar tres aspectos. En primer lugar, garantizar una medición adecuada de las variables de interés, lo cual está ligado a una clara definición conceptual, teniendo en cuentas las implicaciones del apartado anterior. Particularmente, es vital definir el nivel de medición de las variables, dado que la evidencia empírica sobre las sindémias se ha enfocado en analizar variables solo en el nivel individual, lo que discrepa de la teoría porque su objetivo se concentra en la salud de las poblaciones [7]. Los diseños ecológicos y multiniveles serian útiles para superar esta limitación en la evidencia actual.

En segundo lugar, es necesario plantear con claridad el tipo de asociaciones y efectos que se pretenden medir. En el caso de las sindémias va más allá de simples relaciones lineales, dado que en la teoria se pueden abordar asociaciones 
bidireccionales entre enfermedades y problemas en salud, hasta la necesidad de modelos multicausales con nodos interactivos complejos [7]. Para ello, se sugiere realizar análisis de confusión, interacción, moderación de los efectos y técnicas estadisticas avanzadas de inferencia causal [14].

Un tercer aspecto, que ha sido poco medido es el tema de "epidemias causales mutuas". Hay que tener en cuenta los supuestos de causalidad considerando desde el tipo de estudio, en donde es fundamental la temporalidad, hasta los análisis planteados. Se sugiere que futuras investigaciones deberian arrojar evidencia que dé cuenta de la evolución en el tiempo de las epidemias y la incorporación de diseños experimentales para evaluar su interacción [7].

En este sentido, habria que analizar que métodos o diseños de investigación serian los más adecuados. En algunos casos, puede ser necesario aplicar métodos epidemiológicos existentes que tienen en cuenta la complejidad y los múltiples niveles de análisis (multinivel, bayesianos, etc.), mientras que, en otros casos, implicará la aplicación de nuevos métodos de otras disciplinas, como las ciencias sociales.

\section{Conclusión}

La teoría sindémica reconfigura la comprensión histórica convencional de las enfermedades como entidades distintas en la naturaleza, separadas de otras enfermedades e independientes de lo social. La mirada y discusión planteada tiene implicaciones teóricas, metodológicas y prácticas que deben comprenderse mediante la generación de nuevo conocimiento, el cual permita develar formas de abordar la futuras sindémias en diversas áreas del conocimiento y en diferentes contextos sociales o poblaciones.

Ninguna de la evidencia científica sobre la teoría sindémica citada en este artículo es producto de estudios llevados a cabo en el contexto de Latinoamérica. Resultaría relevante poner a prueba la teoría en algunos países de esta región, dado que las tendencias en salud pública en los últimos 25 años han mostrado una evolución rápida de la transición demográfica y epidemiológica, lo que hace que se concentren este tipo de enfermedades en ciertos periodos de tiempo y áreas geográficas, permeado por la influencia de la distribución desigual de los recursos [17].

Por ello, a través de este artículo se hace una invitación a investigadores latinoamericanos para fomentar la aplicación de la teoría sindémica. Resultaría útil a la luz de mejorar la prevención, pronóstico y manejo de las enfermedades y problemas interactuantes. Así como, orientar el diseño e implementación de políticas públicas en salud, dado que las interacciones entre epidemias o problemas de salud pública amplifican la carga de enfermedad. Igualmente, en el área clínica, la teoría sindémica también destaca la necesidad de identificar mejores prácticas para el manejo simultáneo de patologias entrelazadas [3].

Asimismo, la coyuntura actual por COVID-19 demuestra un mayor riesgo de muerte en ciertos grupos poblacionales, en quienes la vulnerabilidad puede estar definida biológica o socialmente. Tal es el caso, de las personas mayores de 60 años y los afroamericanos. En este último tipo de población, la concentración de ciertas enfermedades producto de la exposición a múltiples factores históricos ha generado que experimenten la peor parte de la pandemia actual y la exacerbación de sus consecuencias negativas. La teoría sindémica representa un marco útil para comprender este fenómeno [18].

Finalmente, el futuro de las sindémias estará determinado en gran parte por las actividades humanas, influenciadas por fuerzas sociales a gran escala con impactos nocivos mayores en las poblaciones más vulnerables. Si no se abordan de manera prioritaria estos problemas, las sindémias pueden cobrar un costo enorme en el bienestar poblacional y en la economía de las regiones.

\section{Notas}

\section{Apoyo financiero}

Este trabajo fue financiado parcialmente por el Ministerio de Ciencia, Tecnología e Innovación (MinCiencias) de Colombia, en el marco del Programa de Excelencia Doctoral (Corte 1-2019) concedido a uno de los autores (S. Q. M.). 


\section{Declaración de conflicto de interés}

Los autores declaran que no existen conflictos de intereses relevantes al contenido de este estudio.

\section{Contribuciones de los autores}

Todos los autores han hecho contribuciones sustanciales a la concepción, o diseño, o adquisición, o análisis o interpretación de datos; y redacción del trabajo o revisión crítica; y aprobación final de la versión para publicación.

\section{Disponibilidad de datos y responsabilidad por los resultados}

Todos los autores declaran que han tenido pleno acceso a los datos obtenidos y asumen la plena responsabilidad de la integridad de estos resultados.

\section{Referencias}

1. Mendenhall E. Syndemics: a new path for global health research. Lancet. 2017; 389(10072):889-91. https://doi. org/10.1016/s0140-6736(17)30602-5

2. Singer M, Bulled N, Ostrach B, Mendenhall E. Syndemics and the biosocial conception of health. Lancet. 2017: 389(10072):941-50. http://dx.doi.org/10.1016/ S0140-6736(17)30003-X

3. Singer M. Introduction to Syndemics: a Critical Systems Approach to Public and Community Health. 1st ed. San Francisco (CA): Jossey-Bass; 2009.

4. Nikiphorou E, Lempp H, Kohrt BA. Treatment failure in inflammatory arthritis: Time to think about syndemics? Rheumatol (Oxford). 2019; 58(9):1526-33. https://doi. org/10.1093/rheumatology/kez222

5. Stall R, Coulter RWS, Friedman MR, Plankey MW. Commentary on "Syndemics of psychosocial problems and HIV risk: A systematic review of empirical tests of the disease interaction concept" by A. Tsai and B. Burns. Soc Sci Med. 2015; 145:129-31. http://dx.doi. org/10.1016/j.socscimed.2015.07.016

6. Singer M. A dose of drugs, a touch of violence, a case of AIDS: conceptualizing the SAVA Syndemic. Free Inquiry in Creative Sociology. 1996; 24(2):99-110.

7. Tsai AC, Mendenhall E, Trostle JA, Kawachi I. Co-occurring epidemics, syndemics, and population health. Lancet. 2017:389(10072):978-82. http://dx.doi. org/10.1016/S0140-6736(17)30403-8
8. NCD Risk Factor Collaboration (NCD-RisC). Worldwide trends in blood pressure from 1975 to 2015: a pooled analysis of 1479 population-based measurement studies with $19 \cdot 1$ million participants. Lancet. 2017; 389(10064):37-55. http://dx.doi.org/10.1016/S01406736(16)31919-5

9. Organización Mundial de la Salud. Informe mundial sobre el paludismo 2018. 2018; Disponible en: https://www.who.int/malaria/media/world-malaria-report-2018/es/\#La carga de malaria global y regional en números

10. Eze IC, Bassa FK, Essé C, Koné S, Acka F, Laubhouet-Koffi V, et al. Epidemiological links between malaria parasitaemia and hypertension: findings from a population-based survey in rural Côte d'Ivoire. J Hypertens. 2019; 37(7):1384-92. https://dx.doi.org/10.1097\%2FHJH.0000000000002071

11. Etyang AO, Smeeth L, Cruickshank JK, Scott JAG. The malaria-high blood pressure hypothesis. Circ Res. 2016; 119(1):36-40. https://doi.org/10.1161/circresaha.116.308763

12. Gallego-Delgado J, Rodriguez A. Malaria and hypertension. Another co-evolutionary adaptation? Front Cell Infect Microbiol. 2014:4:121. https://doi.org/10.3389/ fcimb.2014.00121

13. Gallego-Delgado J, Walther T, Rodriguez A. The High Blood Pressure-Malaria Protection Hypothesis. Circ Res. 2016;119(10):1071-5. https://doi.org/10.1161/ circresaha.116.309602

14. Hernán MA, Robins JM. Causal Inference: What If. Boca Raton: Chapman \& Hall/CRC, forthcoming; 2020.

15. Phelan JC, Link BG, Tehranifar P. Social Conditions as Fundamental Causes of Health Inequalities: Theory, Evidence, and Policy Implications. J Health Soc Behav. 2010; 51(Suppl):S28-40. https://doi. org/10.1177/0022146510383498

16. Cañon-Montañez W, Rodriguez-Acelas AL. Use of Causal Diagrams for Nursing Research: a Tool for Application in Epidemiological Studies. Invest Educ Enferm. 2019:37(1):e01. https://doi.org/10.17533/udea.iee.v37n1e01

17. Organización Panamericana de la Salud. Indicadores básicos 2019: Tendencias de la salud en las Américas 2019; https://iris.paho.org/bitstream/handle/10665.2/51543/9789275321287_spa.pdf? sequence $=7 n$

18. Poteat T, Millett G, Nelson LE, Beyrer C. Understanding COVID-19 risks and vulnerabilities among black communities in America: the lethal force of syndemics. Ann Epidemiol. 2020; 47:1-3. https://doi.org/10.1016/i. annepidem.2020.05.004

\section{Silvia Quiroz Mena}

Doctoranda en Epidemiologia por la Universidad de Antioquia (UdeA), Medellin, Colombia. 


\section{Wilson Cañon Montañez}

Doctor en Epidemiologia por la Universidade Federal do Rio Grande do Sul (UFRGS); profesor asociado de la Universidad de Antioquia (UdeA). Medellín, Colombia.

\section{Dirección:}

Sílvia Quiroz Mena

Universidad de Antioquia,

Cl. 64 \#53-09, 050010

Medellín, Antioquía, Colombia. 